Published in final edited form as:

Gastroenterology. 2009 May ; 136(5): 1585-1592. doi:10.1053/j.gastro.2009.01.050.

\title{
Heritability of Nonalcoholic Fatty Liver Disease
}

\author{
Jeffrey B. Schwimmer ${ }^{1,2}$, Manuel A. Celedon ${ }^{1}$, Joel E. Lavine ${ }^{1,2}$, Rany Salem ${ }^{3}$, Nzali \\ Campbell $^{3}$, Nicholas J. Schork ${ }^{3}$, Masoud Shiehmorteza ${ }^{4}$, Takeshi Yokoo ${ }^{4}$, Alyssa Chavez ${ }^{4}$, \\ Michael S. Middleton ${ }^{4}$, and Claude B. Sirlin ${ }^{4}$ \\ ${ }^{1}$ Division of Gastroenterology, Hepatology, and Nutrition, Department of Pediatrics University of \\ California, San Diego School of Medicine, San Diego, California \\ 2 Department of Gastroenterology, Rady Children's Hospital San Diego, San Diego, California \\ ${ }^{3}$ Scripps Genomic Medicine and The Scripps Translational Sciences Institute, Scripps Health \\ and The Scripps Research Institute, La Jolla, California \\ ${ }^{4}$ Liver Imaging Group, Department of Radiology, University of California, San Diego School of \\ Medicine, San Diego, California
}

\section{Abstract}

Background \& Aims-Nonalcoholic fatty liver disease (NAFLD) is the most common chronic liver disease in the United States. The etiology is believed to be multi-factorial with a substantial genetic component; however, the heritability of NAFLD is undetermined. Therefore, a familial aggregation study was performed to test the hypothesis that NAFLD is highly heritable.

Methods-Overweight children with biopsy-proven NAFLD and overweight children without NAFLD served as probands. Family members were studied including magnetic resonance imaging to quantify liver fat fraction. Fatty liver was defined as a liver fat fraction $25 \%$. Etiologies for fatty liver other than NAFLD were excluded. Narrow-sense heritability estimates for fatty liver (dichotomous) and fat fraction (continuous) were calculated using variance components analysis adjusted for covariate effects.

Results-Fatty liver was present in $17 \%$ of siblings and $37 \%$ of parents of overweight children without NAFLD. Fatty liver was significantly more common in siblings (59\%) and parents (78\%) of children with NAFLD. Liver fat fraction was correlated with body mass index (BMI), although the correlation was significantly stronger for families of children with NAFLD than those without NAFLD. Adjusted for age, sex, race, and BMI, heritability of fatty liver was 1.000 and of liver fat fraction 0.386 .

Conclusion-Family members of children with NAFLD should be considered at high risk for NAFLD. These data suggest that familial factors are a major determinant of whether an individual has NAFLD. Studies examining the complex relations between genes and environment in the development and progression of NAFLD are warranted.

\section{Keywords}

magnetic resonance; genetics; family; obesity; fatty liver

Correspondence: Jeffrey B. Schwimmer, M.D. Division of Gastroenterology, Hepatology, and Nutrition, Department of Pediatrics, University of California, San Diego; 200 West Arbor Drive, San Diego, CA 92103. Phone: (619) 543-7544. Fax: (619) 543-7537. jschwimmer@ucsd.edu.

Conflict of Interests: no conflicts of interest exist 


\section{INTRODUCTION}

Nonalcoholic fatty liver disease (NAFLD) is the most common chronic liver disease in adults and children in the developed world. Although obesity is a strong risk factor, the condition of obesity alone is not sufficient to produce NAFLD. There are examples of obese, even morbidly obese, individuals who do not have NAFLD as well as examples of NAFLD occurring in individuals of normal weight. ${ }^{1-5}$ A major unanswered question is what underlies the difference between people who develop NAFLD and those who do not.

One explanation for variation in the development of NAFLD may be heredity, as supported by two lines of reasoning. First, clinical case series have shown familial clustering of NAFLD. ${ }^{6,7}$ A retrospective case series noted that many patients were from families with multiple subjects demonstrating NAFLD. ${ }^{7}$ Second, there are racial and ethnic differences in the prevalence of NAFLD. ${ }^{1,8}$ Among patients of comparable body mass index and insulin resistance, NAFLD is much more likely to occur in Hispanic Americans than among African Americans. ${ }^{1,}{ }^{9-12}$ Although environmental risk factors are likely to influence the development of NAFLD, aspects of the observed variation in NAFLD phenotypic expression in persons with similar metabolic risk factors strongly implicates a genetic contribution.

An important step in deciding whether or not to pursue a genetic linkage or association study for identifying specific genetic variations that might influence NAFLD susceptibility is to determine the heritability of the NAFLD phenotype. ${ }^{13}$ Heritability estimates consider the fraction of the total variation exhibited by a phenotype that can be attributed to genetic factors, and rely on the comparison of the phenotype across related and unrelated individuals. Such estimates can be confounded by shared environmental factors that exist among related individuals and are usually environmentally context-specific. Estimates of heritability among individuals exclusively from one environment might be different from those among individuals from a different environment. ${ }^{14}$ Despite this caveat, heritability estimates are used routinely to assess the likelihood that a phenotype has identifiable genetic determinants. Estimates of the heritability of NAFLD are lacking in large part due to the methodological challenge of accurately determining the phenotype in terms of the presence or absence of NAFLD in each family member. Advances in magnetic resonance imaging (MRI) make accurate noninvasive evaluation feasible for both children and adults. The aim of this study was to estimate the heritability of NAFLD. A familial aggregation study was performed using MRI to assess hepatic steatosis in families of overweight children with and without NAFLD to test the hypothesis that NAFLD is a heritable condition.

\section{METHODS}

\section{Subject Selection}

Probands-Because most children with NAFLD are overweight or obese, all probands were required to be overweight or obese. ${ }^{15}$ Probands were further selected on the basis of the presence or absence of NAFLD. The diagnosis of NAFLD was based upon: 1) liver biopsy with at least $5 \%$ or greater of hepatocytes containing macrovesicular fat ${ }^{16}$, and 2 ) exclusion of other causes of chronic liver disease including hepatitis B (hepatitis B surface antigen), hepatitis $\mathrm{C}$ (hepatitis $\mathrm{C}$ antibody), alpha- 1 antitrypsin deficiency (serum alpha-1 antitrypsin level and histology), autoimmune hepatitis (antinuclear antibody, anti-smooth muscle antibody, and histology), Wilson's disease (serum ceruloplasmin), drug toxicity, total parenteral nutrition, chronic alcohol intake (clinical history), and chronic disease (cystic fibrosis, HIV, celiac disease, type 1 diabetes mellitus). The absence of NAFLD was based upon a liver MRI with a hepatic fat fraction $<5 \%$. 
Family Members-First, second, and third degree family members of probands were recruited. To better ensure that all subjects could follow the study protocol, the minimum age for family members was set at 8 years, with no upper age limit. Exclusion criteria were: 1) inability to complete a MRI evaluation (claustrophobia, metal implants, or body circumference greater than the imaging chamber); 2) potential reasons for hepatic steatosis other than NAFLD including medication (amiodarone, glucocorticoids, L-asparaginase, valproic acid), chronic disease (cystic fibrosis, HIV, hepatitis C, Wilson's disease, celiac disease, type 1 diabetes mellitus), and chronic alcohol consumption; and 3) pregnancy.

The protocol was approved by the institutional review board of the University of California, San Diego. All adult subjects provided written informed consent. The parent(s) of all subjects $<18$ years of age provided written informed consent for their children. Written assent was obtained from all children age 8 to 17 .

\section{Clinical Assessment}

Subjects made a fasting visit to the General Clinical Research Center. Age and sex were recorded. Participants self-identified race and ethnicity. Height and weight were measured with the subjects standing and wearing light clothing without shoes to the nearest $0.1 \mathrm{~cm}$ and $0.1 \mathrm{~kg}$, respectively. Body mass index (BMI) was calculated as the weight $(\mathrm{kg})$ divided by the height $(\mathrm{m})$ squared. Children were classified based upon BMI percentile as normal weight (BMI percentile between the $5^{\text {th }}$ and $84^{\text {th }}$ percentile), overweight (BMI percentile between the $85^{\text {th }}$ and $94^{\text {th }}$ percentile) or obese (BMI percentile $\geq$ the $95^{\text {th }}$ percentile). ${ }^{17}$ Adults were classified based upon BMI as normal weight (BMI 18.5 to 24.9), overweight (BMI 25.0 to 29.9), or obese (BMI = 30.0). Subjects were instructed to fast overnight for 12 hours prior to phlebotomy. Fasting laboratory assays performed in the clinical chemistry laboratory included glucose, insulin, HDL-cholesterol, triglycerides, alanine (ALT) and aspartate (AST) aminotransferase. ALT > $30 \mathrm{U} / \mathrm{L}$ was considered abnormal. Diabetes was defined as a pre-existing clinical diagnosis of type 2 diabetes and/or fasting blood glucose $\geq$ $126 \mathrm{mg} / \mathrm{dL}$.

\section{Determination of Fatty Liver}

Hepatic fat content was measured non-invasively using magnetic resonance (MR) imaging with a modified Dixon technique. ${ }^{18}$ Subjects were scanned supine with a phased-array coil centered over the liver at 1.5T (Siemens Symphony, Siemens Medical Systems, Erlangen, Germany). Multi-slice two-dimensional spoiled gradient recalled echo images were obtained in the axial plane. Field of view was adjusted to individual body habitus. To minimize T1 effects, a low flip angle $\left(10^{\circ}\right)$ was used with a repetition time of 122 msec. ${ }^{19-21}$ To assess fat-water signal interference effects and correct for T2* effects, ${ }_{19}, 21,22$ six echoes were obtained at serial out-of-phase and in-phase echo times $(2.3,4.6,6.9,9.2,11.5,13.8 \mathrm{msec})$ during a single breath-hold. Other imaging parameters were 8 -mm slice thickness, $500-\mathrm{Hz} /$ pixel receiver bandwidth, one signal average, and 256×160-256 matrix.

For each subject, images at each of the six echo times were reviewed on a diagnostic-quality picture archiving and communication system digital monitor. A representative axial slice of the liver was selected. Avoiding organ boundaries, imaging artifacts, major vessels and bile ducts, a single elliptical region of interest (ROI), approximately $300-400 \mathrm{~mm}^{2}$, was manually selected within the liver parenchyma and automatically propagated to images at each of the six echo times. Image registration was not necessary as images at each echo time were acquired during the same breath hold and were already co-localized. The average signal intensity within each ROI was measured and recorded. 
Imaging ROI values were analyzed using MATLAB (The MathWorks, Natick, MA). As described by Bydder, the measured signal intensity variation was modeled as a function of echo time. ${ }^{21}$ This method models the interference between fat-water and fat-fat while also correcting for exponential T2* decay. From the model, the fractional fat content in the liver was calculated as the ratio of fat proton density to the sum of the fat and water proton densities, and expressed as a percentage. Liver fat fraction $25 \%$ was defined as fatty liver. A radiologist reviewed all images.

\section{Data Analysis and Heritability Calculation}

Data were expressed as means \pm standard deviations or frequency and percentage.

Categorical variables were compared using Fisher's exact test and continuous variables with Student's $t$-test. Pearson correlation coefficients were used to assess the strength of the relationships between continuous variables. Differences between correlation coefficients were assessed with standard Fisher z-transform based tests. All hypothesis tests were twotailed. Significance was defined at $a=0.05$.

Narrow sense heritability $\left(\mathrm{h}^{2}\right)$ estimates for continuous traits (liver fat fraction, BMI, glucose, triglycerides) and dichotomous traits (fatty liver, obesity, diabetes) were calculated using the suite of variance component-based statistical models and methods implemented in the Sequential Oligogenic Linkage Analysis Routines (SOLAR, Version 4.1.5, Southwest Foundation for Biomedical Research, San Antonio, TX). The range of possible values for $\mathrm{h}^{2}$ is from 0.0 (not heritable) to 1.0 (complete heritability). The variance component models for estimating heritability in SOLAR are linear models that account for the dependencies in the phenotypes among the related individuals in such a way as to partition variation in the phenotype into that variation attributable to the genetic relationships of the subjects and that attributable to other factors. Heritability was based on residual variance after adjusting for covariates (age, sex, race, ethnicity, BMI). Analyses of dichotomous or otherwise nonnormally distributed phenotypes assessed in a traditional variance component modeling framework may inflate type I error rates. ${ }^{23}$, therefore we used the feature in SOLAR that models dichotomous and non-normally distributed traits on the multivariate t-distribution. ${ }^{24}$ In addition, we corrected for participant ascertainment by conditioning on proband phenotypes. ${ }^{25}$

\section{RESULTS}

\section{Description of Probands}

Details for the probands are shown in Table 1. There were 33 children with biopsy-proven NAFLD and 11 children without NAFLD. Age and BMI did not differ between groups. The distribution of overweight and obesity was also the same for both groups: overweight $18 \%$ and obese $82 \%$. The racial and ethnic distribution for children with NAFLD was WhiteHispanic 23/33 (70\%), White-non-Hispanic 7/33 (21\%), and Native American IndianHispanic 3/33 (9\%). For children without NAFLD, the racial and ethnic distribution was White-Hispanic 6/12 (50\%), White-non-Hispanic 2/12 (17\%), Native American Indian Hispanic 3/12 (25\%), Pacific Islander-non-Hispanic 1/12(8\%). Probands with NAFLD had a significantly ( $\mathrm{p}<0.001$ ) higher mean ALT and AST than overweight children without NAFLD. ALT was elevated in 31 of 33 (94\%) children with NAFLD and in 3 of 11 (27\%) children without NAFLD ( $\mathrm{p}<0.001)$. All children with NAFLD had MR-measured liver fat fraction $>5 \%$. Children with NAFLD had a significantly $(\mathrm{p}<0.001)$ higher mean MRmeasured liver fat fraction of $18.1 \%$ compared to $1.6 \%$ for children without NAFLD. 


\section{Description of Family Members}

A total of 152 non-proband family members were studied. The median family size studied was 4 people (range 2 to 12). Family members of children with NAFLD included 29 siblings, 55 parents, and $272^{\text {nd }}$ or $3^{\text {rd }}$ degree relatives. Family members of children without NAFLD included 12 siblings, 19 parents, and $102^{\text {nd }}$ or $3^{\text {rd }}$ degree relatives. Details for the siblings and parents of probands with and without NAFLD are shown in Table 1.

The mean values of the laboratory parameters tested were not significantly different between siblings of children with NAFLD and siblings of children without NAFLD. Serum triglycerides and fasting insulin were significantly $(\mathrm{p}<0.05)$ higher in parents of children with NAFLD than in parents of children without NAFLD. ALT was elevated in 30 of 55 $(55 \%)$ parents of children with NAFLD and in 7 of $19(37 \%)$ parents of children without NAFLD $(p=0.29)$. Diabetes was present in 12 of $55(22 \%)$ parents of children with NAFLD and in 2 of 12 (17\%) parents of children without NAFLD ( $p=0.99)$.

\section{Assessment of Fatty Liver}

MR-measured liver fat fraction was significantly $(\mathrm{p}<0.01)$ higher in siblings $(9.3 \%)$ and parents (14.0\%) of children with NAFLD than siblings (2.7\%) and parents (7\%) of children without NAFLD. For all participants combined including probands, liver fat fraction was significantly $(\mathrm{p}<0.01)$ correlated $(\mathrm{r}=0.54)$ with BMI. As shown in Figure 1 , the correlation between liver fat fraction and BMI was strong in families of children with NAFLD and mild in families of children without NAFLD. The difference in the strength of correlation was significant $(\mathrm{p}=0.029)$.

Fatty liver was present in 59\% (17 of 29) of the siblings and 78\% (43 of 55) of the parents of children with NAFLD. The frequency of fatty liver was significantly $(\mathrm{p}<0.01)$ lower in the siblings (17\%, 2 of 12) and the parents (37\%, 7 of 19) of children without NAFLD. As highlighted by the family pedigree shown in figure 2, fatty liver was present in 20 of 111 (18\%) of family members of children with NAFLD despite being non-obese and having a normal ALT. In family members with fatty liver, mean ALT was mildly elevated $(45 \pm 38$ $\mathrm{U} / \mathrm{L})$ but significantly $(\mathrm{p}<0.01)$ higher than in family members without fatty liver $(27 \pm 21$ $\mathrm{U} / \mathrm{L}$ ). Among family members with fatty liver, the mean liver fat fraction was $15.4 \pm 9 \%$.

Previously unrecognized cirrhosis was detected by MRI in 2 family members of children with NAFLD. Both cases were subsequently confirmed by clinical evaluation including biopsy. The first case was a 62 year-old grandmother with a history of type 2 diabetes and mild liver chemistry elevation (ALT $47 \mathrm{U} / \mathrm{L}$, AST $42 \mathrm{U} / \mathrm{L}$ ). She had previously been told that she had mild fatty liver based upon her serum aminotransferase values. MRI evaluation revealed a liver fat fraction of 7\%, a shrunken liver, and the presence of esophageal varices. The second case was a 39 -year-old father with no known medical problems. He had a BMI of $33.8 \mathrm{Kg} / \mathrm{m}^{2}$. Serum ALT was $62 \mathrm{U} / \mathrm{L}$ and AST was $70 \mathrm{U} / \mathrm{L}$. Liver MRI revealed a hepatic fat fraction of $24 \%$ along with an enlarged caudate lobe and lobulated, nodular liver margins consistent with cirrhosis.

\section{Estimate of heritability}

As shown in Table 2, in the unadjusted model, NAFLD was significantly $(\mathrm{p}<0.001)$ and highly heritable $\left(\mathrm{h}^{2}=0.850\right.$ (SE 0.325)). Using regression-like adjustments for age, gender, race and BMI, the estimated heritability of NAFLD increased to the boundary or maximum value of 1.0 ( $\mathrm{p}<0.0001)$. When assessed as a continuous measure, the heritability for liver fat fraction was $0.581(\mathrm{p}=0.0001)$. After adjustment for age, sex, race and BMI, the heritability estimate for liver fat fraction was attenuated but retained significance $\left(\mathrm{h}^{2}=\right.$ $0.386, \mathrm{p}<0.05)$. Both the continuous measure of BMI and the dichotomous measure of 
obesity also had high heritability. Serum triglycerides were significantly heritable but at a lower level. Neither the diagnosis of diabetes nor serum glucose as a continuous variable demonstrated significant heritability in this study sample.

\section{DISCUSSION}

We performed a familial aggregation study of fatty liver in overweight children with and without NAFLD. In family members, the presence or absence of fatty liver was documented using MRI. Clinical history and laboratory testing were applied to exclude participants with alternate explanations for fatty liver other than NAFLD. The main finding was that liver fat fraction and the condition of fatty liver are heritable traits. The estimate for the heritability of the fatty liver as a dichotomous trait reached the boundary value of 1.0, thus we were not able to determine the exact value for the heritability of fatty liver, but can confidently conclude that the heritability is high.

Earlier evidence for the familial nature of NAFLD came from retrospective case series. Struben et. al. reviewed a clinical database of 124 patients with NASH and 174 with cryptogenic cirrhosis and identified 32 patients who reported a family history of fatty liver and/or cryptogenic cirrhosis. They reviewed the medical records of a total of 10 family members from 8 of the 32 patients with a positive family history and noted the co-existence of NASH and/or cryptogenic cirrhosis in seven of eight kindreds studied. ${ }^{6}$ Willner and colleagues reviewed the charts of 90 patients with a diagnosis of NASH at the University of Tennessee and the Medical University of South Carolina. ${ }^{7}$ The investigators noted that 16 of the 90 patients came from families with 2 or more patients with NASH. Notably cirrhosis was observed in 7 of these 9 families. A case series from Japan described 3 families each with 2 people with biopsy-proven NASH. ${ }^{26}$ In addition to familial aggregation of NAFLD, the phenotypic features associated with NAFLD may also be more prevalent in families with NAFLD. A study of 20 adults with NAFLD and 20 controls without NAFLD, demonstrated that insulin resistance was more prevalent in first degree relatives of patients with NAFLD than those without NAFLD. ${ }^{27}$

The frequency of fatty liver seen in the siblings and parents of overweight children without NAFLD was consistent with population-based estimates. The prevalence of fatty liver in children in the County of San Diego was estimated to be $9.6 \%$ overall and $17.3 \%$ for adolescents age 15 to $19 .{ }^{1}$ For adults age 30 to 65 , the prevalence of hepatic steatosis was estimated to be $34 \%$ in Dallas County. ${ }^{8}$ In sharp contrast, family members of children with NAFLD appear to be at high risk as the majority of both siblings and parents of children with NAFLD were shown to have hepatic steatosis. Targeted screening may be warranted. Moreover, a demonstration of the familial nature of the disease may serve to enhance efforts at modifying the family environment towards increased physical activity and healthier dietary choices. Of additional concern was the detection of 2 cases of unrecognized cirrhosis out of the 33 families with a child with NAFLD studied. Primary care physicians, endocrinologists, gastroenterologists, hepatologists, and pediatricians should consider the possibility of advanced liver disease in the absence of any symptoms.

A novel finding in the current study was the interaction between BMI and familial factors on liver fat fraction. In families of children with NAFLD (i.e., the families at higher risk for fatty liver), the severity of steatosis as determined by the MRI-measured fat fraction was strongly correlated with BMI. In contrast, in families of children without NAFLD (i.e., the families at lower risk for fatty liver), the correlation between BMI and liver fat fraction was weaker. This difference helps to explain why the adjustment for BMI attenuated the heritability estimate for liver fat fraction as a continuous trait but, by reducing noise, increased the heritability estimate for fatty liver as a dichotomous trait. Moreover, these 
observations suggest that BMI is a strong determinant of steatosis severity in those with a heritable susceptibility to fatty liver but not in those without a heritable susceptibility and helps to strengthen the argument that familial factors contribute to the development of hepatic steatosis.

Establishing the heritability of a trait is considered a first step towards the identification of specific genes that influence that trait. Although the strength of the heritability of a trait does not always translate into the ease with which genes can be identified, it does provide support that genes contribute to the phenotypic expression of that trait. It is likely that fatty liver that begins early in life has a stronger genetic component than fatty liver that does not develop until adulthood. Once heritability has been established, a logical next step is to pursue the identification of specific genes that influence the trait via linkage and association analysis. ${ }^{13}$

The accumulation of fat, largely composed of triglyceride, in the hepatocyte is the essential first step in the development of NAFLD. The pathogenesis of steatosis is likely multifactorial, involving both complex genetic and environmental factors that regulate lipid metabolism and the flux of fatty acids to, within and from the hepatocyte. ${ }^{28,}{ }^{29}$ Putative candidate genes include those involved in processes that regulate hepatic lipid metabolism and the flux of fatty acids to the liver from the adipocyte. Free fatty acid in the liver is metabolized by one of two pathways: oxidation to generate ATP or esterification to produce triacylglycerides. Triglycerides are incorporated into VLDL particles for export or stored within hepatocytes. Therefore, hepatic steatosis can be caused by any combination of factors that increase the hepatocyte free fatty acid pool or that decrease mitochondrial $\beta$-oxidation, peroxisomal $\gamma$-oxidation, or VLDL synthesis/secretion. Association studies of single nucleotide polymorphisms as a risk factor for NAFLD offer promising findings for genes related to: (1) the magnitude and pattern of fat deposition, (2) hepatic lipid export, and (3) inflammation and oxidant stress. ${ }^{30-36}$ Similarly, studies of gene expression in hepatic steatosis have shown upregulation of genes involved in lipid metabolism. ${ }^{37-39}$ The studies to date are not definitive because of small sample sizes, the complex issue of appropriate control groups and the lack of replication samples. These data along with the current study support the planning and execution of large scale studies examining genetic factors contributing to NAFLD.

A strength of this study was the use of magnetic resonance imaging, as this allowed for objective and precise determination of hepatic fat fraction. Studies based upon less robust measures for case definition, such as liver chemistry or ultrasonography, are subject to greater error. The use of a diverse study population was notable, especially the inclusion of a large number of Hispanic families because they are at greater risk for NAFLD than nonHispanics. However, this emphasis may limit the generalizability of these findings to nonHispanic families. Another limitation was the lack of liver biopsies in the family members studied. A design including biopsy would be required to determine the hereditability of a sub-phenotype of NAFLD such as steatohepatitis or advanced fibrosis, but is not feasible for a family study which requires the use of a method that can be applied to all family members. Heritability estimates are influenced by shared environmental factors. In order to further separate environmental from genetic causes, future studies will need to collect information on factors including nutrition and activity.

The public health burden of NAFLD is considerable and likely to increase over time. Understanding the genetic and environmental factors that contribute to NAFLD prevalence and severity has important implications for clinical care and public health. The current data demonstrate for the first time that NAFLD is a highly heritable condition. Family members of children with NAFLD should be considered at high risk for NAFLD, even in the absence of obesity or elevated serum aminotransferases. The current data suggest that familial factors 
are a major determinant of whether an individual has NAFLD. Further family studies will help unravel the complex interactions between genes and environment in the development of NAFLD.

\section{Acknowledgments}

Financial Support: This work was funded in part by grants from the National Institutes of Health including R21 DK71486 from the National Institute of Diabetes and Digestive and Kidney Diseases, P60 MD00220 for the San Diego EXPORT Center from the National Center of Minority Health and Health Disparities, and M01 RR000827 from the National Center for Research Resources for the General Clinical Research Center at UCSD. RS and NJS are supported in part by Scripps Genomic Medicine and The Scripps Translational Sciences Institute. The contents of this work are solely the responsibility of the authors and do not necessarily represent the official views of the National Institutes of Health.

\section{Abbreviations}

NAFLD nonalcoholic fatty liver disease

NASH nonalcoholic steatohepatitis

BMI body mass index

MRI magnetic resonance imaging

FF fat fraction

\section{References}

1. Schwimmer JB, Deutsch R, Kahen T, Lavine JE, Stanley C, Behling C. Prevalence of Fatty Liver in Children and Adolescents. Pediatrics. 2006; 118:1388-1393. [PubMed: 17015527]

2. Beymer C, Kowdley KV, Larson A, Edmonson P, Dellinger EP, Flum DR. Prevalence and Predictors of Asymptomatic Liver Disease in Patients Undergoing Gastric Bypass Surgery. Arch Surg. 2003; 138:1240-1244. [PubMed: 14609874]

3. Xanthakos S, Miles L, Bucuvalas J, Daniels S, Garcia V, Inge T. Histologic spectrum of nonalcoholic fatty liver disease in morbidly obese adolescents. Clinical Gastro Hep. 2006; 4:2262332.

4. Kim HJ, Lee KE, Kim DJ, et al. Metabolic significance of nonalcoholic fatty liver disease in nonobese, nondiabetic adults. Arch Intern Med. 2004; 164:2169-2175. [PubMed: 15505132]

5. Nobili V, Marcellini M, Devito R, et al. NAFLD in children: a prospective clinical-pathological study and effect of lifestyle advice. Hepatology. 2006; 44:458-465. [PubMed: 16871574]

6. Struben VM, Hespenheide EE, Caldwell SH. Nonalcoholic steatohepatitis and cryptogenic cirrhosis within kindreds. Am J Med. 2000; 108:9-13. [PubMed: 11059435]

7. Willner IR, Waters B, Patil SR, Reuben A, Morelli J, Riely CA. Ninety patients with nonalcoholic steatohepatitis: insulin resistance, familial tendency, and severity of disease. Am J Gastroenterol. 2001; 96:2957-2961. [PubMed: 11693332]

8. Browning JD, Szczepaniak LS, Dobbins R, et al. Prevalence of hepatic steatosis in an urban population in the United States: impact of ethnicity. Hepatology. 2004; 40:1387-1395. [PubMed: 15565570]

9. Browning JD, Kumar KS, Saboorian MH, Thiele DL. Ethnic differences in the prevalence of cryptogenic cirrhosis. Am J Gastroenterol. 2004; 99:292-298. [PubMed: 15046220]

10. Szczepaniak LS, Nurenberg P, Leonard D, et al. Magnetic resonance spectroscopy to measure hepatic triglyceride content: prevalence of hepatic steatosis in the general population. Am J Physiol Endocrinol Metab. 2005; 288:E462-E468. [PubMed: 15339742]

11. Schwimmer JB, McGreal N, Deutsch R, Finegold M, Lavine JE. The Influence of gender, race, and ethnicity on suspected fatty liver in obese adolescents. Pediatrics. 2005; 115:e561-e565. [PubMed: 15867021] 
12. Louthan MV, Theriot JA, Zimmerman E, Stutts JT, McClain CJ. Decreased prevalence of nonalcoholic fatty liver disease in black obese children. J Pediatr Gastroenterol Nutr. 2005; 41:426-429. [PubMed: 16205510]

13. Khoury, MJ.; Beaty, TH.; Cohen, BH. Fundamentals of genetic epidemiology. New York: Oxford University Press; 1993.

14. Vitzthum VJ. A number no greater than the sum of its parts: the use and abuse of heritability. Hum Biol. 2003; 75:539-558. [PubMed: 14655876]

15. Schwimmer JB, Behling C, Newbury R, et al. Histopathology of pediatric nonalcoholic fatty liver disease. Hepatology. 2005; 42:641-648. [PubMed: 16116629]

16. Brunt EM. Nonalcoholic steatohepatitis: definition and pathology. Semin Liver Dis. 2001; 21:316. [PubMed: 11296695]

17. Committee on Prevention of Obesity in Children and Youth. Preventing Childhood Obesity Health in the Balance. Washington, D.C: The National Academies Press; 2005.

18. Dixon WT. Simple proton spectroscopic imaging. Radiology. 1984; 153:189-194. [PubMed: 6089263]

19. Hussain HK, Chenevert TL, Londy FJ, et al. Hepatic fat fraction: MR imaging for quantitative measurement and display--early experience. Radiology. 2005; 237:1048-1055. [PubMed: 16237138]

20. Liu CY, McKenzie CA, Yu H, Brittain JH, Reeder SB. Fat quantification with IDEAL gradient echo imaging: correction of bias from T(1) and noise. Magn Reson Med. 2007; 58:354-364. [PubMed: 17654578]

21. Bydder M, Yokoo T, Hamilton G, et al. Relaxation effects in the quantification of fat using gradient echo imaging. Magn Reson Imaging. 2008; 26:347-359. [PubMed: 18093781]

22. Yu H, McKenzie CA, Shimakawa A, et al. Multiecho reconstruction for simultaneous water-fat decomposition and T2* estimation. J Magn Reson Imaging. 2007; 26:1153-1161. [PubMed: 17896369]

23. Allison DB, Neale MC, Zannolli R, Schork NJ, Amos CI, Blangero J. Testing the robustness of the likelihood-ratio test in a variance-component quantitative-trait loci-mapping procedure. Am J Hum Genet. 1999; 65:531-544. [PubMed: 10417295]

24. Lange K, Little RJA, Taylor JMG. Robust statistical inference using the t distribution. Journal of the American Statistical Association. 1989; 84:881-896.

25. Boehnke M, Lange K. Ascertainment and goodness of fit of variance component models for pedigree data. Prog Clin Biol Res. 1984; 147:173-192. [PubMed: 6547532]

26. Tokushige K, Yatsuji S, Hashimoto E, et al. Familial aggregation in patients with non-alcoholic steatohepatitis. Intern Med. 2008; 47:405-410. [PubMed: 18310971]

27. Abdelmalek MF, Liu C, Shuster J, Nelson DR, Asal NR. Familial aggregation of insulin resistance in first-degree relatives of patients with nonalcoholic fatty liver disease. Clin Gastroenterol Hepatol. 2006; 4:1162-1169. [PubMed: 16901766]

28. Chitturi S, Farrell GC. Etiopathogenesis of nonalcoholic steatohepatitis. Semin Liver Dis. 2001; 21:27-41. [PubMed: 11296694]

29. Day CP. The potential role of genes in nonalcoholic fatty liver disease. Clin Liver Dis. 2004; 8:673-691. [PubMed: 15331069]

30. Shima Y, Tsukada T, Nakanishi K, Ohta H. Association of Trp64Arg mutation of the B3adrenergic receptor with fatty liver and mild glucose intolerance in Japanese subjects. Clinica Chimica Acta. 1998; 274:167-176.

31. Iwamoto N, Ogawa Y, Kajihara S, et al. Gln27Glu beta2-adrenergic receptor variant is associated with hypertriglyceridemia and the development of fatty liver. Clin Chim Acta. 2001; 314:85-91. [PubMed: 11718682]

32. Valenti L, Fracanzani AL, Dongiovanni P, et al. Tumor necrosis factor alpha promoter polymorphisms and insulin resistance in nonalcoholic fatty liver disease. Gastroenterology. 2002; 122:274-280. [PubMed: 11832442]

33. Namikawa C, Shu-Ping Z, Vyselaar JR, et al. Polymorphisms of microsomal triglyceride transfer protein gene and manganese superoxide dismutase gene in non-alcoholic steatohepatitis. J Hepatol. 2004; 40:781-786. [PubMed: 15094225] 
34. Nozaki Y, Saibara T, Nemoto Y, et al. Polymorphisms of Interleukin-1B and B3-Adrenergic Receptor in Japanese Patients with Nonalcoholic Steatohepatitis. Alcohol Clin Exp Res. 2004; 28:106S-110S. [PubMed: 15318095]

35. Song J, da Costa KA, Fischer LM, et al. Polymorphism of the PEMT gene and susceptibility to nonalcoholic fatty liver disease (NAFLD). FASEB. 2005; 19:1266-1271.

36. Brun P, Castagliuolo I, Floreani AR, et al. Increased risk of NASH in patients with the C(-159)T polymorphism in the CD14 gene promoter region. Gut. 2006; 55:1212. [PubMed: 16849359]

37. Westerbacka J, Kolak M, Kiviluoto T, et al. Genes involved in fatty acid partitioning and binding, lipolysis, monocyte/macrophage recruitment, and inflammation are overexpressed in the human fatty liver of insulin-resistant subjects. Diabetes. 2007; 56:2759-2765. [PubMed: 17704301]

38. Greco D, Kotronen A, Westerbacka J, et al. Gene expression in human NAFLD. Am J Physiol Gastrointest Liver Physiol. 2008; 294:G1281-1287. [PubMed: 18388185]

39. Higuchi N, Kato M, Shundo Y, et al. Liver X receptor in cooperation with SREBP-1c is a major lipid synthesis regulator in nonalcoholic fatty liver disease. Hepatol Res. 2008; 38:1122-1129. [PubMed: 18684130] 
A.

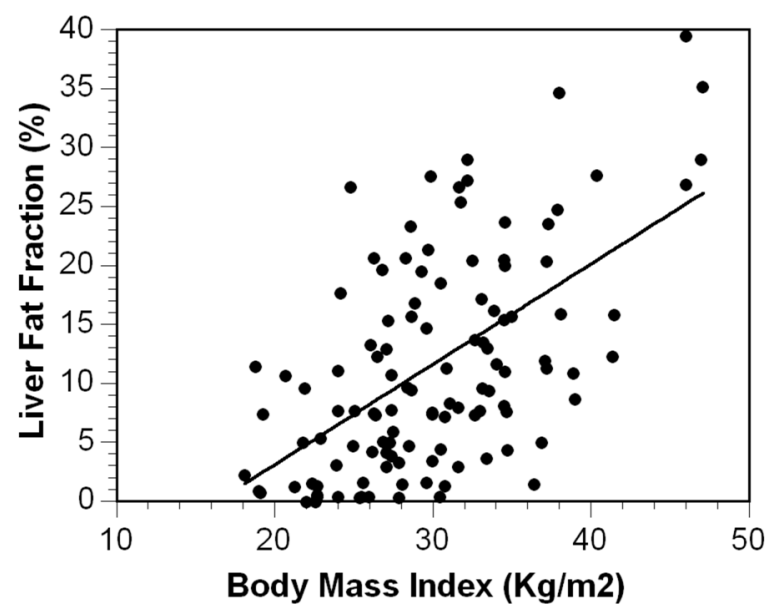

B.

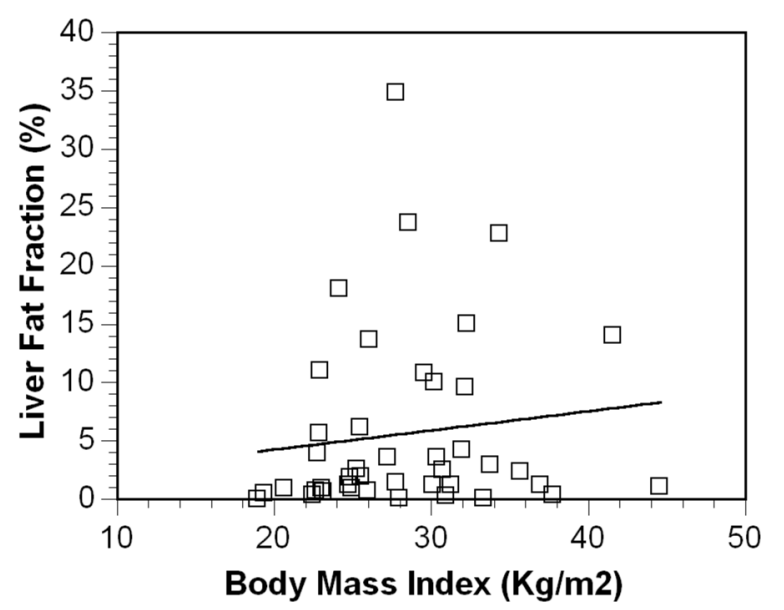

Figure 1. Correlation of BMI and Liver Fat Fraction

Panel A: the correlation between BMI and liver fat fraction was high $\left(r^{2}=0.61 ; p<0.01\right)$ in family members of children with NAFLD $(\mathbf{O})$. Panel B: the correlation $\left(\mathrm{r}^{2}=0.26 ; \mathrm{p}<0.05\right)$ between BMI and liver fat fraction was low in family members of children without NAFLD ( ). The difference in correlation was significant $(\mathrm{p}=0.029)$. 
I

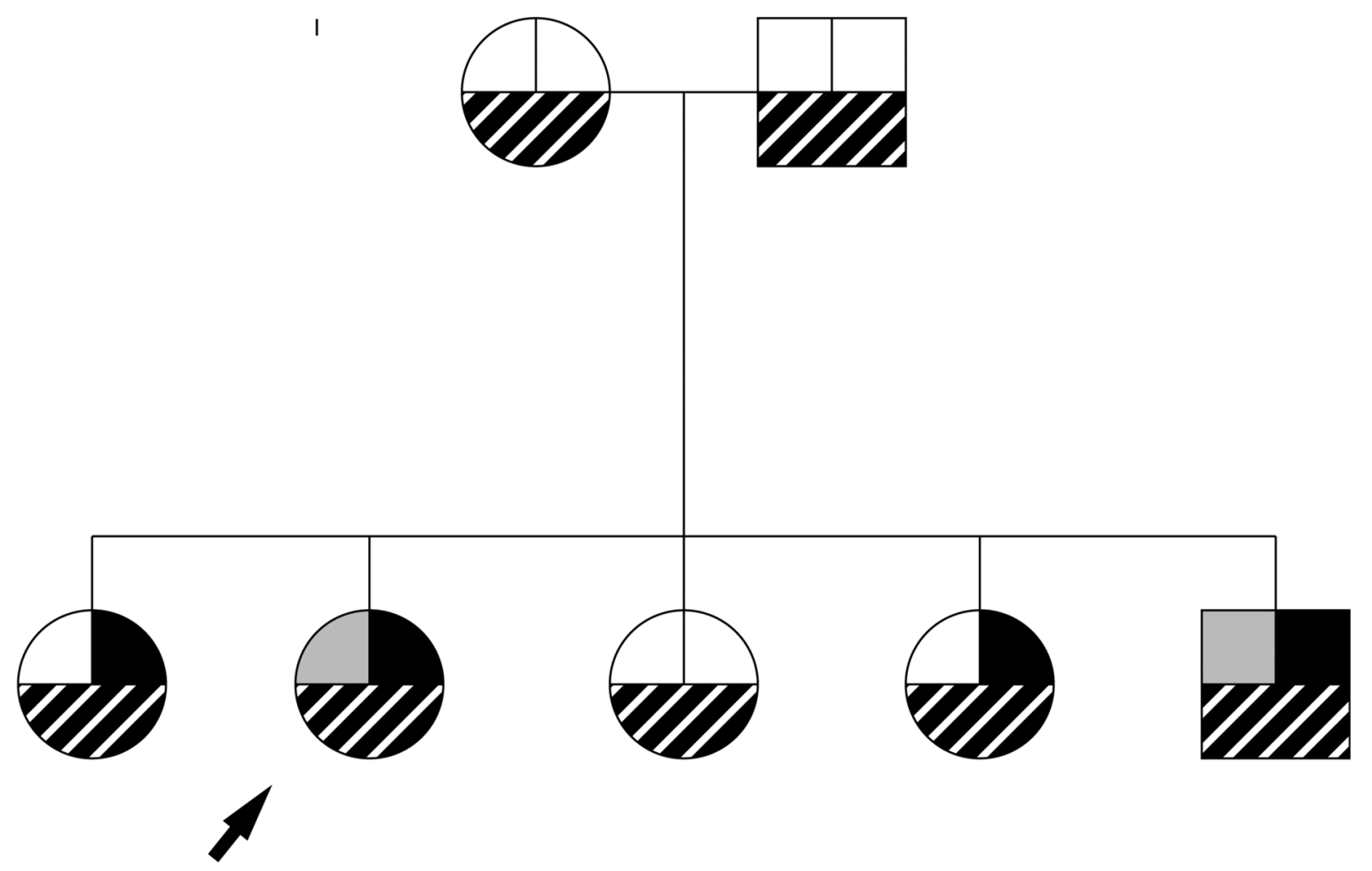

II

Figure 2. Paradigmatic pedigree of a child with NAFLD

The arrow indicates the proband, a girl with biopsy-proven NAFLD. She is shown to be obese (left upper box with grey fill), have an elevated ALT (right upper box with black fill) and to have a liver fat fraction $>5 \%$ (bottom box with striped fill). Her family can be seen to have only 1 other obese individual, 3 other with an elevated ALT, but fatty liver in all sibliings and parents. 


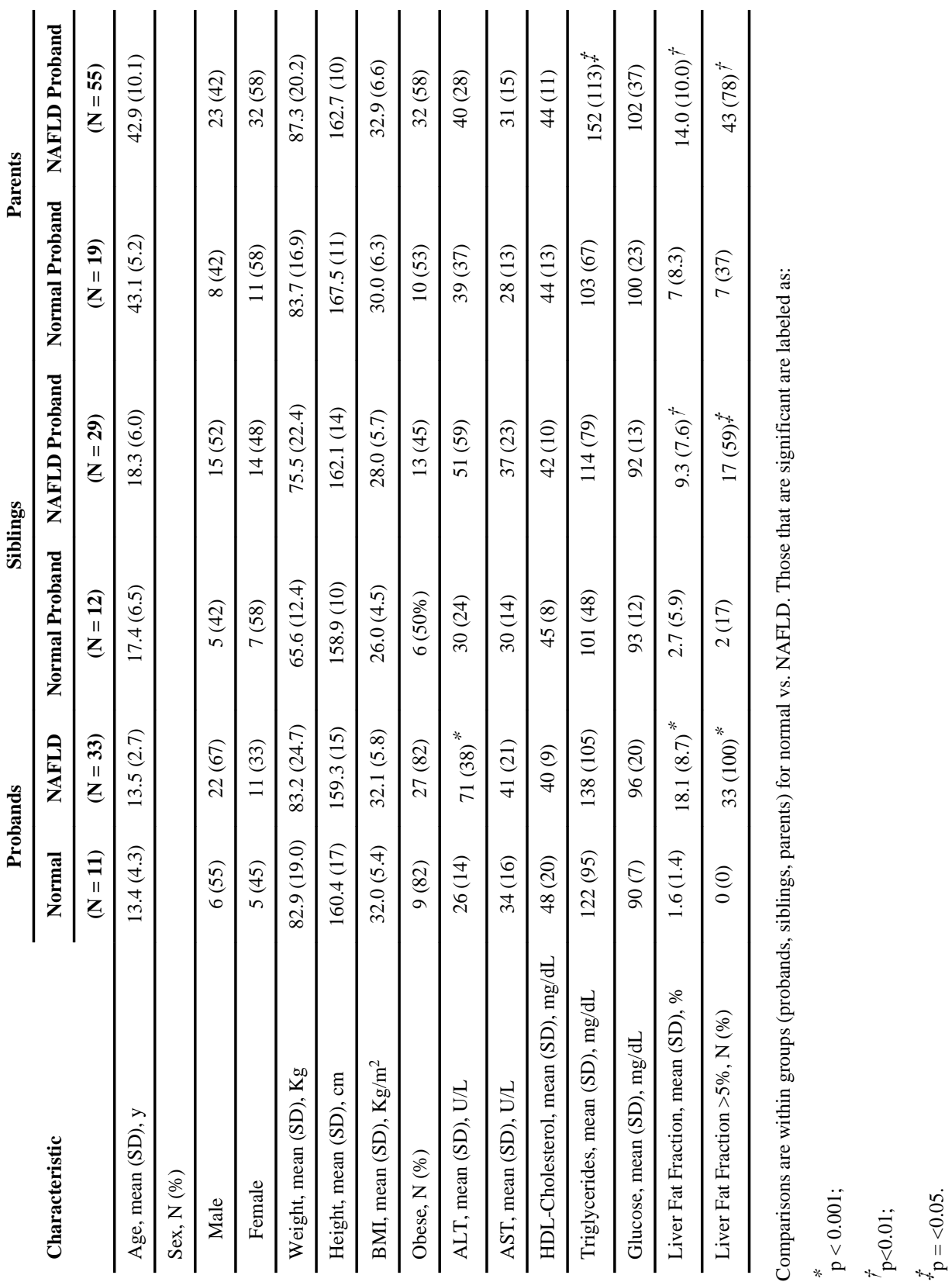




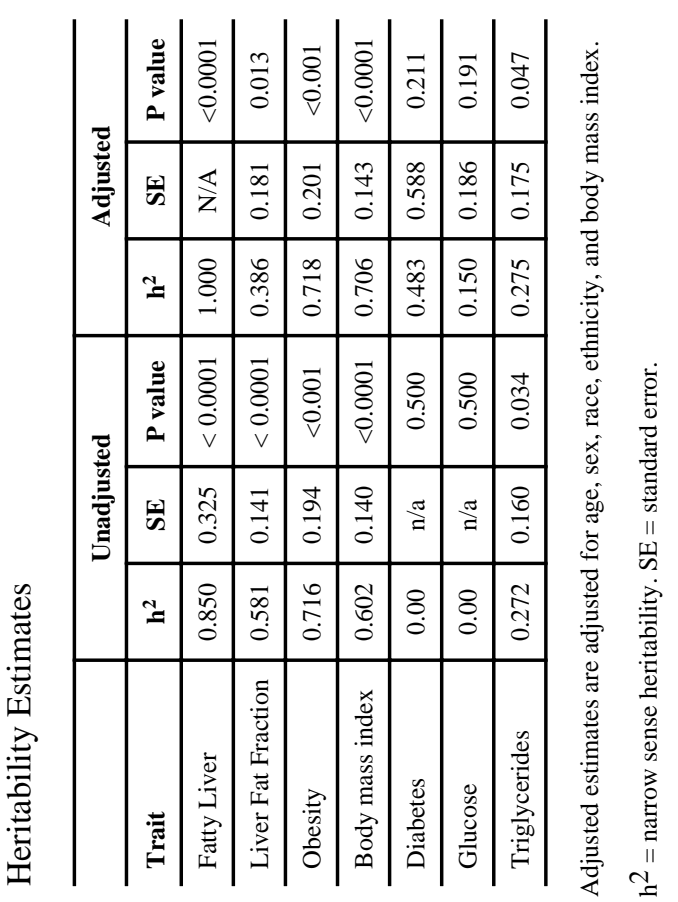

